with either paraphrenic symptoms or delusional depression without any signs of cognitive impairment, at least not in the early stages.

The fact that the clinical classification system has developed without a separate category for these patients should give clinicians considerable cause for concern. In the past, the diagnostic boundaries of Alzheimer's disease have probably been stretched to accommodate these presentations, with concepts such as 'the clinical heterogeneity of Alzheimer's disease' being used to justify such expansions. The more characteristic presentations of Lewy body dementia may now be identified by use of appropriate clinical criteria (McKeith et al, 1992a).

Förstl et al suggest that diffuse Lewy bodies may occur in some cases of Alzheimer's disease as a coincidental phenomena, and cite previous studies which have found a 12-15\% "incidental Lewy body" rate in aged normals. The normal populations examined in these studies had either a psychiatric (Forno, 1969) or neurological bias (Gibb et al, 1989) and therefore reflect an association between Lewy body pathology and central nervous system disease rather than with normal ageing. A Newcastle study found a $2.3 \%$ rate of Lewy body formation in 131 medical and surgical patients with demonstrably normal neurological and mental states (Perry et al, 1990). The coincidental occurrence of Lewy bodies in patients with Alzheimer's disease appears then to be an incomplete explanation for the $12 \%$ Lewy body rate found by the authors in their sample.

The precise nature of the relationship between Lewy body and Alzheimer-type pathologies, and the different clinical syndromes associated with each remains to be established. Until then, clinicians should be aware of the broad spectrum of clinical presentations of cortical Lewy body disease, ranging from delusional states in the absence of marked cognitive impairment to 'typical' Alzheimer-type dementia. Between these extremes the most frequent clinical presentation appears to involve fluctuating cognitive impairment often associated with psychotic features, Parkinsonism, transient disturbance of consciousness and falls, and sensitivity to neuroleptic medication.

FORNo, L. S. (1969) Concentric hyalin inclusions of Lewy type in the brains of elderly persons ( 50 incidental cases): relationship to Parkinsonism. Journal of the American Geriatric Society, 17, $557-575$.

GrbB, W. R. G., MountJoy, C. Q., MANn, D. M. A., et al (1989) A pathological study of the association between Lewy body disease and Alzheimer's disease. Journal of Neurology, Neurosurgery and Psychiatry, 52, 701-708.

McKeith, I. G., Perry, R. H., Fatrbairn, A. F., et al (1992a) Operational criteria for senile dementia of Lewy body type Psychological Medicine, 22, 911-922.
- Fairbairn, A., Perry, R., et al (1992b) Neuroleptic sensitivity in patients with senile dementia of Lewy body type. Britsh Medical Journal, 305, 673-678.

Perry, R. H., Irving, D. \& Tomlinson, B. E. (1990) Lewy body prevalence in the ageing brain: relationship to neuropsychiatric disorders, Alzheimer type pathology and catecholaminergic nuclei. Journal of the Neurological Sciences, 100, 223-233.

IAN MCKEITH

ANDREW FAIRBAIRN

RUTH BRIEL

RICHARD HARRISON

Nick TASKER

Brighton Clinic

Newcastle Mental Health (NHS) Trust

Westgate Road

Newcastle upon Tyne NE4 6BE

\section{Human sleep, sleep loss, and behaviour}

SIR: I would like to comment on the paper by Horne (Journal, March 1993, 162, 413-419) in which he provided intriguing evidence for a link between prefrontal dysfunction, human slow-wave sleep (hSWS) and depression. One line of argument focused on the neuropsychological effects of total sleep deprivation (TSD) in normal subjects pointing to some reversible dysfunction of the prefrontal cortex (PFC).

However, there is an additional aspect of TSD which was not explicitly mentioned by the author: TSD also has a brief but significant antidepressant effect in about $50 \%$ of depressed patients. Hypofrontality in the left dorsolateral PFC measured with the single photon emission computerised tomography (SPECT) method is present in this subset of depressed patients already before TSD, and, different from normal subjects, this hypofrontality is not increased by TSD. There is even a tendency for TSD to reduce hypofrontality, and this trend is more obvious in TSD responders than in non-responders(Ebert et al, 1991). This means that TSD may have different effects in normal subjects and depressed patients, but on the other side it strengthens the author's view that hypofrontality may only be a secondary effect of cognitive underactivation in depression.

The effects of TSD in depression, however, do not argue for a proposed theory that a decrease of hSWS in depressed patients (with or without frontal impairment) is part of the aetiology of the disorder because, if an impairment of hSWS was responsible for depression, TSD should not act as an antidepressant, and relapse after TSD should not occur in sleep after TSD with the highest hSWS rebound.

Instead, we may have to look for a theory taking into account that "sleep itself with its related neurobiological mechanisms is the cause of depression and wakefulness is the antidepressant agent". With the 
evidence given, some of these proposed depressiogenic sleep-related mechanisms may indeed occur during the hSWS period. An obvious possibility for such a mechanism may be the physiologically altered balance of catecholaminergic-cholinergic neurotransmitters with consecutive changes of receptor sensitivities during hSWS. If future research gave more evidence for this hypothesis, it should be asked next whether altered hSWS in depression is a source of primary dysfunction, or whether the decrease is secondary to depression to avoid the depressiogenic effects of 'normal' hSWS.

There is another possible source of hSWS involvement in the origin of depression: hSWS reduces the heat load and dampens hypermetabolic states, and it may be of importance that those depressive patients react best to TSD who have higher nightly temperatures than controls or TSD non-responders (Wehr, 1990).

As mentioned by Dr Horne, it is not necessarily the dorsolateral PFC which is involved in hSWS related depression, but the chain of events leading to depressiogenic hSWS may start in other brain regions, for example the gyrus cingularis and the fronto-orbital cortex. Thus, in previous SPECT and positron emission tomographic studies these two structures were found to be hyperactive in TSD responders before TSD, compared with non-responders and controls, and hypermetabolism was suppressed by TSD (Ebert et al, 1991; Wu et al, 1992). With the cingulate cortex being also hyperactive during rapideye-movement sleep, these findings point to sleep stages other than hSWS as being involved in the origin of depression. However, the possible link between fronto-orbital and cingulate hyperfusion and dorso-lateral prefrontal hyperperfusion demonstrated with SPECT (Ebert et al, 1991) still supports Dr Horne's view of hSWS and PFC involvement in depression, and, additionally, both activity patterns are not contradictory to a theory of a catecholaminergic-cholinergic imbalance in a subset of depressed patients.

Ebert, D. Feistel, H. \& BAROCKA, A. (1991) Effects of sleep deprivation on the limbic system and the frontal lobes in affective disorders-A HMPAO SPECT Study. Neuroimaging, 40, 247-251.

WEHR, T. A. (1990) Effects of sleep and wakefulness on depression and mania. In Sleep and Biological Rhythms (eds J. Montplaisir \& R. Godbout), pp. 42-86. London: Oxford University Press.

Wu, J. C., Gilln, J. C., Buchsbaum, M., et al (1992) Effect of sleep deprivation on brain metabolism of depressed patients. American Journal of Psychiatry, 149, 538-543.

University of Erlangen

D. EBERT

Schwabachanlage 6-10

8520 Erlangen

Germany

\section{A HUNDRED YEARS AGO}

\section{Broadmoor criminal lunatic asylum}

Dr Nicolson's annual report for the year ending 1891 upon the Broadmoor Criminal Lunatic Asylum shows that the asylum population included 336 persons who had committed homicide, 205 of whom were men and 131 women. These cases of homicide consisted of 313 cases of murder and 23 of manslaughter. The admissions to the hospital included 41 men and 15 women during the year. Of these 33 were "Queen's pleasure" inmates and the remaining 23 consisted of insane convicts. The report points out that although there were in all 539 afflicted people in the asylum suffering from various degrees of insanity yet at no period during the whole year had it been found necessary to rebuke any member of the staff for imprudence in dealing with those under his charge. This is a very satisfactory statement. The report also mentions that no inmate had succeeded in effecting an escape, and that it had not been found necessary during the year to have recourse to mech- anical restrain in any form. The facts and figures stated above afford ample evidence of the nature of the organisation and of the excellence of the arrangements made at Broadmoor for the care of criminal lunatics. On Friday, the 3rd inst., the staff and attendants of the asylum enjoyed their annual entertainment. In the course of some complimentary remarks to the members of the staff Dr Nicolson expressed himself as being highly pleased with the efficient assistance which they had rendered during the past year. We take the opportunity of congratulating Dr Nicolson himself and his assistant medical officers, Dr Issac, Dr Noott and Mr W. E. Miles, on the able manner in which the onerous and difficult duties appertaining to the management of the institution are carried out.

\section{Reference}

Lancet, 11 February 1893, 311. 